\title{
Neumonía adquirida en la comunidad asociada a tabaquismo pasivo en pediatría
}

\section{Community-acquired pneumonia associated with passive smoking in pediatrics}

\section{Neumonia adquirida na comunidade associada ao tabaquismo pasivo na pediatria}

\author{
Nancy A. Sorroza-Rojas I \\ nancysorroza@uees.edu.ec \\ José P. Barberan-Torres II \\ pbarberan@uees.edu.ec \\ Nancy V. Cajas-Flores III \\ ncajasf@uees.edu.ec
}

Homero E. Jinez-Jinez IV

hejinezjinez@hotmail.com
Edith E. López-Montanero V elopezm@uees.edu.ec

Jesús E. Rodríguez-Villacis VI jesusrodriguez@uees.edu.ec

Recibido: 30 de enero de 2017 * Corregido: 20 de febrero de 2017 * Aceptado: 20 junio de 2017

\footnotetext{
I. Universidad de Especialidades Espíritu Santo, Samborondón, Ecuador.

II. Universidad de Especialidades Espíritu Santo, Samborondón, Ecuador.

III. Universidad de Especialidades Espíritu Santo, Samborondón, Ecuador.

IV. Universidad de Especialidades Espíritu Santo, Samborondón, Ecuador.

v. Universidad de Especialidades Espíritu Santo, Samborondón, Ecuador.

VI. Universidad de Especialidades Espíritu Santo, Samborondón, Ecuador.
} 


\section{Resumen}

En esta investigación se aborda los efectos del tabaquismo en las vías respiratorias de los pacientes pediátricos, siendo expuestos al humo del cigarrilo como fumadores pasivos, trayendo como consecuencia padecimientos como la neumonía. Avalado en diversos estudios de expertos en la materia de Iberoamerica, se estudia todas las implicaciones que conlleva inhalar todos los tóxicos del tabaco a tan corta edad. El trabajo arroja como resultados una fuerte vinculación con los padres fumadores y los ambientes en donde se desenvuelven los infantes con dichas enfermedades respiratorias. También se definen unas recomendaciones para la prevención de dichas patologías.

Palabras Claves: Tabaquismo, fumador pasivo, vías respiratorias, pediátrico y neumonía. 


\begin{abstract}
This research addresses the effects of smoking in the respiratory tract of pediatric patients, being exposed to cigarette smoke as passive smokers, resulting in conditions such as pneumonia. Endorsed in several studies of experts in the field of Iberoamerica, we study all the implications of inhaling all tobacco toxins at such a young age. The work shows as a result a strong link with the smoking parents and the environments in which the infants develop with said respiratory diseases. Some recommendations for the prevention of said pathologies are also defined.
\end{abstract}

Key Words: Smoking, passive smoking, respiratory, pediatric and pneumonia. 


\section{Introducción.}

El tabaquismo es el principal factor de riesgo de muerte prematura prevenible en el mundo (Wald, 1996). El consumo activo y pasivo de tabaco tiene efectos dañinos sobre múltiples órganos, pero es especialmente nocivos en el sistema respiratorio (Sherman, 1992). Es importante definir que el fumador pasivo es la persona que en un ambiente cerrado inhala humo de tabaco procedente de otros fumadores.

Esta situación puede reproducir en el no fumador la situación del fumador activo que consume entre cuatro a 10 cigarrillos. Esto es debido a que el humo que aspira el fumador pasivo o involuntario contiene una concentración de determinados componentes tóxicos muy superior a la del humo que aspira el fumador directamente del cigarrillo (EPA, 2016).

La inhalación activa o pasiva a humo de tabaco ocasiona los siguientes cambios morfológicos en el pulmón: inflamación y fibrosis peribronquiolar, alteración de la estructura y función del epitelio alveolar, engrosamiento de la íntima vascular y destrucción de alvéolos. Otras consecuencias son las alteraciones funcionales asociadas al tabaquismo, que incluyen la disminución del clearance mucociliar de sustancias inhaladas, favorece la adherencia de microorganismos a la mucosa respiratoria y cambios en la permeabilidad alvéolo-capilar secundario al aumento en el número y actividad de las células inflamatorias en el pulmón (Saldias et al, 2007).

La exposición de la vía aérea a microorganismos es constante, pero el pulmón sano dispone de suficientes mecanismos de defensa para prevenir su invasión. Cualquier fallo de estos mecanismos puede producir diseminación de microorganismos en el árbol bronquial y desencadenar 
neumonía adquirida en la comunidad (NAC), que sigue siendo una causa importante de morbimortalidad en los países desarrollados (Piatti, 1997).

\section{Materiales y Métodos.}

La investigación documental será el método que se usará en esta investigación y puede definirse como una estrategia en la que se observa y reflexiona sistemáticamente sobre realidades teóricas y empíricas usando para ello diferentes tipos de documentos donde se indaga, interpreta, presenta datos e información sobre un tema determinado de cualquier ciencia, utilizando para ello, métodos e instrumentos que tiene como finalidad obtener resultados que pueden ser base para el desarrollo de la creación científica

También se puede comprender en las siguientes características:

- La recolección, selección, análisis y presentación de información coherente a partir del uso de documentos.

- La realización de una recopilación adecuada de datos e información que permiten redescubrir hechos, sugerir problemas, orientar hacia otras fuentes de investigación, orientar formas para elaborar instrumentos de investigación, elaborar hipótesis, etc.

- Considerarse como parte fundamental de un proceso de investigación científica, mucho más amplio y acabado.

- Realizase en forma ordenada y con objetivos precisos, con la finalidad de ser base para la construcción de conocimientos. 
- El uso de diferentes técnicas e instrumentos para la localización y clasificación de datos, análisis de documentos y de contenidos (Martínez, 2002)

En el presente texto se hizo uso de material bibliográfico no experimental para llevar a cabo el estudio. Fundamentados en artículos científicos de varios expertos de Iberoamérica en vías respiratorias para determinar la influencia del tabaquismo en patalogías neumonologicas en pacientes pediátricos, los cuales son expuestos al humo del cigarrillo como fumadores pasivos. Enfocado en la neumonía, determinando la relevancia que tiene las toxinas del tabaco en el origen de la misma y recomendaciones para evitarlo en dichos pacientes.

\section{Resultados.}

En diversos estudios durante las últimas décadas en Iberoamérica, expertos en las vías respiratorias han coincidido en el efecto perjudcial que representa para los niños ser expuestos al humo del cigarrillo como fumadores pasivos. En dichos estudios los investigadores han obtenidos los siguientes resultados:

- El tabaquismo activo y pasivo continúa siendo un problema grave de salud pública en el medio nacional y se asocia a mayor riesgo de desarrollar infecciones respiratorias, debido a los efectos nocivos de la nicotina y otros componentes del tabaco sobre la estructura y función del sistema respiratorio y el sistema inmune del huésped. (Saldias,2007)

- Los pacientes pediátricos son más vulnerables debido a que fisiológicamente tienen una frecuencia respiratoria más elevada, igualmente inhalan más contaminantes por Kg. de peso que adultos y el metabolismo más lento de la cotinina (Willers, 1995) 
- Abrir una ventana o la restricción de fumar en una habitación específica ofrece poca protección contra la exposición al cigarrillo. Investigadores han encontrado que el humo de un cigarrillo puede permanecer en una habitación durante un máximo de dos horas y media, incluso con una abierta. (Ott Wr, 2003)

- El tabaquismo pasivo es un factor de riesgo importante para la salud, especialmente durante la época pediátrica

\section{Conclusiones.}

- Existe una relación bien establecida entre el tabaquismo parental y el riesgo de infecciones respiratorias bajas en niños. Hay estudios realizados que muestran que los hijos pequeños de padres fumadores tienen el doble riesgo de padecer infecciones respiratorias bajas

- Los hijos de madres fumadoras tienen más ingresos hospitalarios por bronquiolitis y neumonía.

- El humo de tabaco de segunda mano es hasta 10 veces más cargado de sustancias tóxicos que el humo principal.

- La contaminación del cigarrillo puede persistir en alfombras, muebles y paredes. Estos materiales absorben las toxinas que se encuentran en el humo del tabaco y liberan gradualmente de nuevo en el aire, lo que representa un riesgo adicional de exposición (Becquemin, 2010) 


\section{Recomendaciones.}

- En los pacientes que consultan por infecciones respiratorias agudas se debería evaluar el riesgo individual de exposición a humo de tabaco y el equipo de salud debería implementar actividades educativas específicas para intentar controlarlo

- Como medidas preventivas adicionales, se debería recomendar la vacunación antiifluenza y antineumocócica trecevalente conjugada. (Díaz, 2017)

- Una de las tareas prioritarias de los pediatras es conseguir ambientes domésticos libres de humo tabáquico

\section{Bibliografía.}

WALD N J, HACKSHAW A K. Cigarette smoking: an epidemiological overview. Br Med Bull 1996; 52: 3-11.

SHERMAN C B. The health consequences of cigarette smoking. Pulmonary diseases. Med Clin North Am 1992; 76: 355-75

EPA. Respiratory Health Effects of Passive Smoking. Environmental Protection Agency 600/690/006F (pag. 7/10-7/20). Washington DC 2046

G. Piatti,T. Gazzola,L. Allegra. Bacterial adherence in smokers and non-smokers

Pharmacol Res, 36 (1997), pp. 481-484

SALDÍAS P, FERNANDO, MÉNDEZ C, IGNACIO, RAMÍREZ R, DAVID, \& DÍAZ P, ORLANDO. (2007). El riesgo de infecciones respiratorias en el fumador activo y pasivo. Revista chilena de enfermedades respiratorias, 23(3), 179-187.

Willers S. Arch of Environ. Health, 1995. 50(2):130-138

Díaz J. Muñoz M. (2017) Infecciones respiratorias en fumadores. Neumoexpertos en prevención.

Ott WR. Journal of the Air \& Waste Management Association. 2003.

Becquemin. Tobacco Control 2010; 19(4):347-8 
Vol. 4, núm. 1, enero, 2018, pp. 268-279

Neumonía adquirida en la comunidad asociada a tabaquismo pasivo en pediatría

Martínez, S (2002) Guía de apuntes básicos para el docente de la materia de técnicas de investigación en Grupo Emergente de Investigación Oaxaca [En línea] México 